\title{
Pairing of the transfer experiment with the kindling paradigm: A summary of results
}

\author{
JOHN GAITO \\ York University, Downsview M3J 1P3, Ontario, Canada
}

\begin{abstract}
Eighteen experiments pairing the transfer experiment with the kindling paradigm were conducted. The brain homogenate supernatant from rats kindled to clonic convulsions was injected intraperitoneally into naive recipients. Similar material from nonkindled rats was injected into other naive recipients. One, two, two and one-half, and three brain amounts were used. Recipients receiving supernatant from kindled animals were retarded significantly in the development of clonic convulsions for all brain amounts. No clear retardation effect was obtained if the supernatant was injected intracerebrally or if the recipients had reached the convulsion stage.
\end{abstract}

The kindling paradigm (Goddard, McIntyre, \& Leech, 1969) was instituted in this laboratory a few years ago in lieu of learning procedures to determine brain chemical events which accompany behavioral changes. This paradigm involves periodic low-level stimulation via implanted electrodes in a number of brain sites. With repeated stimulation, behavior slowly changes from normal exploration and eventually culminates in clonic convulsions (CC). Using this approach, some investigators (Goddard et al., 1969; McIntyre \& Goddard, 1973) reported positive and/or negative transfer of an intraanimal nature (e.g., from one amygdala to the other). We were interested in determining the possibility of interanimal transfer, i.e., pairing the "kindling effect" with the "transfer experiment" (Gaito, 1971). This paper is concerned with a summary of the data from 18 experiments.

In each experiment, the brain coordinates used for electrode implantation were $.5 \mathrm{~mm}$ posterior to bregma. $4.5 \mathrm{~mm}$ from midline, and $8.5 \mathrm{~mm}$ from skull. Previously, histological analyses indicated that the tip of the electrode was in the amygdala. In all experiments, Wistar-strain male rats (90 to 130 days old at the beginning of the experiment) had bipolar electrodes implanted into the amygdala (Nichrome wire with trimel coating. $.127 \mathrm{~mm}$ in diam, dipped one time in Epoxylite). Electrodes were inserted into the right or left hemisphere according to a random process. All electrodes were inspected for electrical adequacy before insertion. Six or more days after surgery, the kindling procedures began. Experimental rats were stimulated three times daily (with $1 \mathrm{~h}$

Research reported in this paper was supported by the President's NRC Fund (Grant 32) from York University and a grant from the Ontario Society for Crippled Children. In the conduct of these experiments, the following personnel have been involved: the author. Wayne Pelletier, Robert Hopkins, Donna Gaito, Olive Gaito. Stephen Gaito, Paul Sanberg, and Lawrence Scrima. Howard Flock sponsors this paper and takes full editorial responsibility for its content. between trials) by a $60-\mathrm{Hz}$ sine wave of 100 -microA intensity (peak to peak) for $30 \mathrm{sec}$ from a Lafayette sine wave stimulator. A response was scored as a clonic convulsion (CC) only if the convulsion continued after termination of the current. On the 10th CC trial, at 10 min after the initiation of the trial, the rats were sacrificed by decapitation. The brain was rapidly removed; the olfactory bulbs, cerebellum, and brainstem parts below the cerebellum were deleted; the remainder was used for injections. Control donors (C) vere connected to stimulator lead wires but received no stimulation. The Cs were sacrificed and treated in the same manner as the Es.

TheE brains were homogenized together in physiological saline $(2 \mathrm{ml} /$ brain $)$. A similar procedure was used with $\mathrm{C}$ brains. The homogenates were shaken over night at $2^{\circ} \mathrm{C}$ and centrifuged at $20,000 \times \mathrm{g}$ for $1 \mathrm{~h}$. The homogenate supernatant from each of the $E$ and $C$ rats was pooled separately and used for intraperitoneal (IP) injection of specific brain amounts into $\mathrm{E}$ and $\mathrm{C}$ recipients. In some experiments, the supernatants were run through an Amicon ultrafiltration cell to separate fractions at a molecular weight of 500 or 10,000 prior to injection.

Recipient rats were prepared in the same manner as the donors. Twenty-four hours after injection, brain stimulation of the $E$ and $C$ recipients began. All recipients were coded and run by an experimenter who was unaware of the designation of each rat. The number of trials to first CCwas the dependent variable of concern.

Ten experiments involved the evaluation of the effects of brain homogenate supernatant from kindled (i.e., stimulated to CC stage, E) and nonkindled (C) rats on the kindling rats of naive recipients. The results of the first experiments showed that a "retardation effect" was present when rats were injected IP with supernatant from kindled rats, if the amount injected was equivalent to that from two or more brains (Gaito \& Gaito, 1974). Other research 
concerned with the isolation and identification of the molecular species producing the negative transfer suggested that the component had a molecular weight greater than 500 (Gaito, 1975). In these 10 experiments, varying brain amounts were used: one, two, two and one-half, and three brains. The means and number of rats used with each brain amount are shown in Table 1 for these experiments.

A two-factor analysis of variance was performed on these data. With the region of the null hypotheses (Ho) set below a probability level of .05, neither brain amounts nor the interaction of groups with amounts allowed rejection of the respective Ho. However, Ho was rejected for the groups source $(F=12.26$; $\mathrm{df}=1.190 ; \mathrm{p}<.001)$. These results indicated a constant difference between $\mathrm{E}$ and $\mathrm{C}$. The means and number of animals were: $E-14.3,118 ; C-10.2,80$.

Separate regression analyses were performed on $\mathrm{E}$ and $\mathrm{C}$ data to ascertain the regression equation for each group. An orthogonal polynomials procedure was used for this purpose. Because the intervals between brain amounts were not equal, the orthogonal polynomial coefficients for the linear, quadratic, and cubic components were derived by the method suggested by Gaito (1965, 1973). Although the regression equation in this case is of the form $y=\mu+\beta_{1} c_{1}+\beta_{2} c_{2}+\beta_{3} c_{3}$ (where the $\beta$ s are the regression coefficients for each of the three components and the cs are the orthogonal polynomial coefficients), the statistical analyses indicated that none of the estimators (b) of the regression coefticients were significantly different from zero in any analyses. Thus, these results suggest that the regression equations are of the form, $y=\mu$, a straight line equal to the mean. The predicted curves for $E$ and $\mathrm{C}$ are estimated as $\mathrm{y}=\mu_{\mathrm{e}}=14.3$ and $\mathrm{y}=\mu_{\mathrm{c}}=10.2$. The significant groups effect and nonsignificant interaction source in the two-factor analysis is consistent with this interpretation.

These experiments have not provided a reliable estimate for the 0 brain point. However, experimental donors could be used for this purpose. Donors tend to show a value around 10 (Gaito, 1975), which is similar to the mean for the $C$ recipients.

Although the early experiments (Gaito \& Gaito, 1974) suggested that the retardation effect is present only if two or more brain amounts are used for injections. the present analyses over all of the data

Table 1

Mean Number of Trials to First CC

\begin{tabular}{|c|c|c|c|c|c|}
\hline \multirow{4}{*}{$\begin{array}{l}E^{*} \\
C^{*}\end{array}$} & \multicolumn{5}{|c|}{ Brain Amounts } \\
\hline & 1 & \multicolumn{2}{|c|}{2} & 2.5 & 3 \\
\hline & $13.9 \quad(34)$ & 15.0 & (28) & $14.9 \quad(34)$ & $12.9 \quad(22)$ \\
\hline & 12.2 (16) & 8.3 & (13) & $10.5 \quad(33)$ & $9.4 \quad(18)$ \\
\hline
\end{tabular}

${ }^{*}$ Reject Ho below $p$ of .05 . E v's. Cover brain amounts. Number of rats in parentheses. show that the effect also is present for one brain. Obviously, these analyses are more powerful in rejecting null hypotheses (because all of the data are used) than are the separate analyses employed previously.

We can conclude from this set of experiments that the retardation is due to neurochemical changes occurring during kindling or to brain stimulation per se. To differentiate these two possibilities, we need a control group which is stimulated in the amygdala by a frequency which does not produce kindling, e.g., $3 \mathrm{~Hz}$ (Goddard et al., 1969).

These results point in a clear fashion to a retardation effect produced by the supernatant. They are in sharp contrast to results of two other studies conducted in our laboratory. In the one case, intracerebral injections were used (Gaito, 1975). The results tended in the direction of retardation in two of six experiments (statistically significant differences in only one), but overall the means for $E$ and $C$ were similar. However, the mean number trials to first CC was elevated for both $E$ and $C$ animals (E, 16.3. 37 rats; C, 15.6. 37 rats). It is possible that a retardation effect was present but was obscured by increased "noise level" due to the brain injection.

The results also are different than those obtained when recipients have reached the convulsion stage. In this case, in two experiments with 15 pairs of rats, no effect was produced by the supernatant (Gaito, Note 1); both $\mathrm{E}$ and $\mathrm{C}$ rats convulsed immediately when stimulated.

It is uncertain whether the molecular component in the $E$ brain responsible for the retardation effect in the IP experiments is qualitatively or quantitatively different than that in the $C$ brain. The results of a few experiments using ultrafiltration procedures to separate the supernatant according to molecular weight suggest that the responsible chemical has a molecular weight greater than 500 , and probably less than 10,000 . This molecular weight range would include a number of biologically active molecules (e.g., hormones, polypeptides, or proteins with 5 to 100 amino acids, and various other molecules).

A few experiments have been conducted also using thin-layer chromatography in an attempt to detect qualitatively different polypeptide or protein patterns between $E$ and $C$ rats. No differences have been found (Gaito. Note 2). Also no differences are detected with polyacrylamide gel electrophoresis analyses (Gaito et al., 1973).

\section{REFERENCE NOTES}

1. Gaito. J. Lack of retardation with convulsed recipients in the kindling paradigm. Submitted for publication. 1975.

2. Gaito, J. An attempt to identify the interanimal retardation factor in the kindling effect by thin layer chromatography. Submitted for publication, 1975. 


\section{REFERENCES}

Gaito. J. Unequal intervals and unequal n. Psychological Bulletin. $1965,63,125-127$.

Gaito. J. DNA complex and adaptive behavior. Englewood Cliffs. N. J: Prentice-Hall. 1971.

GaITo. J. Introduction to analysis of variance procedures. New York: MSS Information Corp., 1973.

GaIto, J. Further results with pairing of the kindling effect and transfer experiment. Physiological Psychology, 1975, 3, 237.2.39.

Gaito. J., \& Gaito, S. T. Interanimal negative transfer of the kindling effect. Physiological Psychology, 1974, 2, 379-382.
Gaito, J., Hopkins, R. W., \& Pelletier, W. Interanimal transfer and chemical events underlying the kindling effect. Bulletin of the Psychonomic Socicty. 1973, 1, 319-321.

Goddard, G. V., McIntyre, D. C., \& Leech, C. K. A permanent change in brain function resulting from daily electrical stimulation. Experimental Neurology, 1969, 25, 295-330.

MCInTyRE, D., \& Goddard, G. V. Transfer, interference and spontaneous recovery of convulsions kindled from the rat amygdala. Electroencephalography \& Clinical Neurophysiology, $1973,35,533-543$.

(Received for publication October 3, 1975) 